\title{
Constraints on neutrino non-standard interactions from LHC data with large missing transverse momentum
}

\author{
DianYu Liu, ${ }^{a}$ ChuanLe Sun ${ }^{a}$ and Jun Gao ${ }^{a, b}$ \\ ${ }^{a}$ INPAC, Shanghai Key Laboratory for Particle Physics and Cosmology, \\ School of Physics and Astronomy, Shanghai Jiao-Tong University, \\ Shanghai 200240, China \\ ${ }^{b}$ Center for High Energy Physics, Peking University, \\ Beijing 100871, China \\ E-mail: dianyu.liu@sjtu.edu.cn, chlsun60@sjtu.edu.cn, \\ jung49@sjtu.edu.cn
}

ABSTRACT: The possible non-standard interactions (NSIs) of neutrinos with matter plays important role in the global determination of neutrino properties. In our study we select various data sets from LHC measurements at $13 \mathrm{TeV}$ with integrated luminosities of $35 \sim 139 \mathrm{fb}^{-1}$, including production of a single jet, photon, $W / Z$ boson, or charged lepton accompanied with large missing transverse momentum. We derive constraints on neutralcurrent NSIs with quarks imposed by different data sets in a framework of either effective operators or simplified $Z^{\prime}$ models. We use theoretical predictions of productions induced by NSIs at next-to-leading order in QCD matched with parton showering which stabilize the theory predictions and result in more robust constraints. In a simplified $Z^{\prime}$ model we obtain a 95\% CLs upper limit on the conventional NSI strength $\epsilon$ of 0.042 and 0.0028 for a $Z^{\prime}$ mass of 0.2 and $2 \mathrm{TeV}$ respectively. We also discuss possible improvements from future runs of LHC with higher luminosities.

Keywords: Neutrino Physics, Beyond Standard Model

ArXiv EPrint: 2009.06668 


\section{Contents}

1 Introduction 1

2 Model assumptions and constraints 3

2.1 Theoretical setup 3

2.2 Data selection 6

2.3 Constraints for effective operator 8

2.4 Constraints for simplified $Z^{\prime}$ model $\quad 9$

3 Theoretical uncertainties $\quad 11$

$\begin{array}{lll}3.1 & \text { Next-to-leading order QCD corrections } & 11\end{array}$

$\begin{array}{ll}3.2 & \text { Constraints at NLO } \\ \end{array}$

$\begin{array}{lll}4 & \text { LHC combination and projections } & 13\end{array}$

$\begin{array}{llr}5 & \text { Conclusion } & 16\end{array}$

\section{Introduction}

Confirmation on neutrino oscillation in recent decades requires non-vanishing neutrino masses. The effective operator with lowest dimensions that respects Standard Model(SM) gauge symmetry is the dimension-five Weinberg operator. This operator can give rise to neutrino masses, and can be achieved by different UV-complete models depending on the portal particle [1-4]. Many extensions of the SM such as Supersymmetry also introduce dimension-six operators of the form

$$
\mathcal{L}_{\mathrm{NSI}, \mathrm{CC}}=-2 \sqrt{2} G_{F} \epsilon_{\alpha \beta}^{f f^{\prime}, Y}\left(\bar{\nu}_{\alpha} \gamma_{\mu} P_{L} l_{\beta}\right)\left(\bar{f}^{\prime} \gamma^{\mu} P_{Y} f\right)+\text { h.c. }
$$

known as Charged-Current Non-Standard neutrino Interaction(CC NSI) and

$$
\mathcal{L}_{\mathrm{NSI}, \mathrm{NC}}=-2 \sqrt{2} G_{F} \epsilon_{\alpha \beta}^{f, Y}\left(\bar{\nu}_{\alpha} \gamma_{\mu} P_{L} \nu_{\beta}\right)\left(\bar{f} \gamma^{\mu} P_{Y} f\right)+\text { h.c. }
$$

known as Neutral-Current NSI (NC NSI), that was first proposed by Wolfenstein in 1977 to explain the neutrino oscillation [5]. $\epsilon_{\alpha \beta}^{f f^{\prime}, Y}$ and $\epsilon_{\alpha \beta}^{f, Y}$ define the strength of NSIs respectively, $\alpha, \beta \in\{e, \mu, \tau\}, f$ denote charged leptons or quarks and $P_{Y}$ is chiral projection operator $\left(P_{L}\right.$ or $\left.P_{R}\right)$. CC NSI modifies the neutrino production and detection through its effect on processes such as muon decay and inverse beta decay [6-8]. From those processes severe bound can be obtained [9]. NC NSI plays an important role in neutrino oscillation experiments due to modification to the effective Hamiltonian, especially the matter potential $[5,10-$ 16]. This modification further leads to nuisances and degeneracies to the measurement of neutrino oscillation parameters [17-24]. On the contrary to the tight bounds on CC 
NSI, NC NSI is less constrained and has been studied extensively [9, 25]. There are many experiments giving constraints on NSIs such as XENON1T [26], KM3NeT-ORCA [27], IceCube [28, 29], DUNE [30-34], Super-Kamiokande [35] and Borexino Phase II [36].

Long-baseline(LBL) experiments are the next generation neutrino oscillation experiments for their sensitivity to neutrino mass ordering and CP violating phase $\delta_{C P}$ [37-42]. In recent $\mathrm{T} 2 \mathrm{~K}$ measurement, a preference of normal mass ordering and a best-fit value of $\delta_{C P}$ at -1.89 corresponding to this ordering is reported [43]. The incorporation of NC NSI however complicates the analysis by introducing new CP violating sources and parameter degeneracies [44]. The degenerate LMA-Dark solution results in a preference for the inverted mass ordering and therefore an almost total loss of sensitivity thereof [22].

Compared with oscillation experiments, neutrino scattering experiments are complementary for two reasons. First, the parameter degeneracies are broken-down since for scattering experiments, the measured cross section subjects to no periodicity and there is no unobservable overall phase factor from wave functions. This further makes it possible to constrain individual diagonal parameters in the effective Hamiltonian rather than their differences. Second, measurements in oscillation experiments generally depend on the composition of the media, while scattering experiments are less flavor dependent. CHARM and $\mathrm{NuTeV}$ experiments report the ratios of neutral-current and charged-current neutrinonucleon deep-inelastic scattering cross sections [45, 46]. In the presence of NSI, the ratios of cross sections are modified and are constrained by experimental measurements. For NSI induced by heavy mediator with mass larger than the experimental energy scale, bounds on NSI parameters ranging from sub-percent level to a few percent level are obtained by a global fit to data from current oscillation experiments and the two scattering experiments [47], under assumption that NSI affects only up or down quark at a time. Strong constraints on NSI parameters involving $\mu$ and $\tau$ flavors are also obtained. For mediator with mass lower than $\mathcal{O}(\mathrm{GeV})$, the contact-interaction approximation is invalid in deep-inelastic scattering energy range, but yet still work in coherent neutrino-nucleus scattering (CE $\nu \mathrm{NS})$ of which the momentum transfer lies at $\mathcal{O}(10 \mathrm{MeV})$. In this scenario, similar bounds can be set taking advantage of the recent COHERENT measurement [48-57]. These works also make it clear that in combination with data from scattering experiments degeneracies on neutrino parameters can be resolved to some extent.

High-energy colliders can also help with study of NSIs. In previous works, limits on the NSIs from $e^{+} e^{-}$colliders and the LHC are given by [58, 59] and [60-62] respectively. Other new physics searches such as Dark Matter, Supersymmetry have also been studied at LHC [63-75]. LHC offers a unique way to study neutrino physics for neutrino energy larger than $300 \mathrm{GeV}$ [76]. Different from oscillation and other scattering experiments, the flavor of NSI is indistinguishable at LHC. Besides, LHC is sensitive to both vector-like and axial vector like NSI as opposite to oscillation experiments that only the former relates. Thus the LHC experiment plays a further complementary role in searches of NSI [77-79]. Neutrinos produced by NSI at the LHC would leave large unbalanced transverse energy or momentum in detectors. The major irreducible SM backgrounds are from the decay of $W$ and $Z$ bosons to neutrinos. Meanwhile, an underlying theory model, however, is generally needed since in this scenario the validity of effective field theory (EFT) approach 
is no longer guaranteed. Simplified $Z^{\prime}$ models with possible UV-completions have been considered [80-86]. And given that the mass of $Z^{\prime}$ boson is much larger than momentum transfer at LHC, one can come back to the EFT case. It is noted that similar signals can be produced for various models with dark matters at the LHC. To discriminate these two sources, one can add the shape of distribution of missing energy to the data analysis [87]. Also, given consideration that neutrinos are produced along with charged leptons due to the $\mathrm{SU}(2)_{L}$ doublet nature, data from multi-lepton channel can be complemented to give further discrimination [57, 87].

In this paper, we focus on the aforementioned $Z^{\prime}$ model and study constraints on NC NSI parameters based on various measurements at LHC $13 \mathrm{TeV}$ with large missing transverse momentums in the final states. We considered data sets on production of monojet, mono- $W / Z$ boson, mono-lepton and mono-photon recorded by both ATLAS and CMS collaborations. We conclude the CMS mono-jet data imposes the strongest constraints, and a flavor-blind bound of a few per mille has been obtained for $Z^{\prime}$ mass around $2 \mathrm{TeV}$.

The rest of this paper is organized as follows. In section 2, we discuss the model assumptions and the resultant constraints. In section 3, we discuss the theoretical uncertainties. The LHC combination and projections are presented in section 4 , and we conclude in section 5 .

\section{Model assumptions and constraints}

Phenomenologies of neutrino non-standard interactions can span an energy range from $\mathrm{MeV}$ in neutrino oscillations to $\mathrm{TeV}$ at high energy colliders, or even higher in reaction of cosmic neutrinos. Simplified models or descriptions of NSIs are always adopted in various analyses and for easy comparison of constraints from different experiments. We outline the simplified models used in our study and then the constraints obtained with LHC data.

\subsection{Theoretical setup}

It is justified to express the NSI in a model-independent manner using the effective field theory framework in neutrino study at low energies, for example in study of neutrino oscillations. NC NSI between neutrino and matters can be described by dimension-six four fermion operators in the EFT framework as [5, 88, 89]

$$
\mathcal{L}_{\mathrm{NSI}}=-2 \sqrt{2} G_{F} \epsilon_{\alpha \beta}^{f, Y}\left(\bar{\nu}^{\alpha} \gamma_{\mu} P_{L} \nu^{\beta}\right)\left(\bar{f} \gamma^{\mu} P_{Y} f\right),
$$

where $G_{F}$ is the Fermi constant, $\epsilon_{\alpha \beta}$ is the strength of NSIs, $\alpha, \beta$ denotes the lepton flavors $\{e, \mu, \tau\}$, and $f$ can be either charged leptons or quarks. $P_{Y}$ can be $P_{L}$ or $P_{R}$ which are chiral projectors of left-handed and right-handed. In our study we focus on NSI between neutrinos and quarks of both up and down-type $f=\{u, d\}$. In general the above operators can be embedded into a gauge invariant operator from integrating out heavy degree of freedoms of new physics,

$$
-\frac{c}{\Lambda^{2}}\left(\bar{L}_{\alpha} \gamma_{\mu} L_{\beta}\right)\left(\bar{Q}_{Y} \gamma^{\mu} Q_{Y}\right),
$$


where $L$ is the $\mathrm{SU}(2)_{L}$ doublet of leptons, $Q_{Y}=\left\{Q_{L}, u_{R}, d_{R}\right\}$ are $\mathrm{SU}(2)_{L}$ doublet or singlet of quarks. $\Lambda$ is the typical scale of the new physics models and $c$ is the Wilson coefficient. The conventional NSI strength $\epsilon$ can be related to $\Lambda$ as $\epsilon_{\alpha \beta}=c /\left(2 \sqrt{2} G_{F} \Lambda^{2}\right)$. Stringent limits on NSI exist due to various measurements on charged leptons at colliders once the interaction also involves charged leptons as in eq. (2.2), for example see ref. [57] for recent discussions.

At high energies, for instance at the LHC, effects of neutrino NSI may not be simply described by EFT operators since the momentum transfers can be sufficiently high to resolve further dynamics of the new physics. In this study we focus on a simplified model with NSI between neutrinos and quarks induced by $s$-channel exchange of a $Z^{\prime}$ boson. This simplified model is more appropriate than the aforementioned EFT description at high energy regions. The effective Lagrangian of the interactions can be written as [62]

$$
\mathcal{L}_{\mathrm{NSI}}^{Z^{\prime}}=-\left(g_{\nu}^{\alpha \beta} \bar{\nu}_{\alpha} \gamma^{\mu} P_{L} \nu_{\beta}+g_{q}^{Y} \bar{q} \gamma^{\mu} P_{Y} q\right) Z_{\mu}^{\prime}
$$

where $Z_{\mu}^{\prime}$ represents the force mediator with mass $M_{Z^{\prime}}$. We assume the interactions are independent of quark generations, and only contain vector current for simplicity, namely $g_{u}^{L}=g_{u}^{R} \equiv g_{u}$ and $g_{d}^{L}=g_{d}^{R} \equiv g_{d}$. At low energies or for a $Z^{\prime}$ boson with sufficiently large mass the $s$-channel $Z^{\prime}$ model can be matched onto the EFT representation defined in eq. (2.1) with

$$
\epsilon_{\alpha \beta}^{u(d), V} \equiv \frac{g_{\nu}^{\alpha \beta} g_{u(d)}}{2 \sqrt{2} G_{F} M_{Z^{\prime}}^{2}}
$$

where the superscript $V$ indicates a vector-current form on the matter side in eq. (2.1). There are many new physics models on ultraviolet completion of neutrino NSI such as Zee Model [90] and One-Loop LQ Model [91, 92].

In this study we utilize experimental measurements on signature with large missing transverse momentum at the LHC to constrain neutrino NSI. We select recent ATLAS and CMS data sets on production of mono-jet, mono-photon, mono- $W / Z$ and mono-lepton. The representative Feynman diagrams of these processes as induced by neutrino NSI are shown in figure 1 for the $Z^{\prime}$ model at tree level. We include the interference with SM production as well. At the LHC one will not be able to identify the flavor of neutrinos in the final states. We introduce $\epsilon^{u(d)} \equiv \sum_{\alpha, \beta}\left|\epsilon_{\alpha \beta}^{u(d), V}\right|^{2}$ summed over all neutrino flavors. It is understood that in case of $Z^{\prime}$ model above couplings are constructed out from the couplings with $Z^{\prime}$ as in eq. (2.4). The NSI contributions to cross sections at LHC thus are directly sensitive to $\epsilon^{u(d)}$ with which we set the constraint. ${ }^{1}$ The LHC measurements on NSI are complementary to those from neutrino oscillations in the sense that they probe absolute values of the couplings rather than differences of couplings of different flavors. We present constraints on NSI in both frameworks of EFT and simplified $Z^{\prime}$ model. It is understood that the former one equals the later constraint with sufficiently large $M_{Z^{\prime}}$.

\footnotetext{
${ }^{1}$ In the actual calculation we assume only $\epsilon_{e e}^{u(d), V}$ are non-zero and derive the constraint. However, since the interference effects between NSI and SM interactions are small, one can translate the same constraint to $\epsilon^{u(d)}$ as a good approximation.
} 


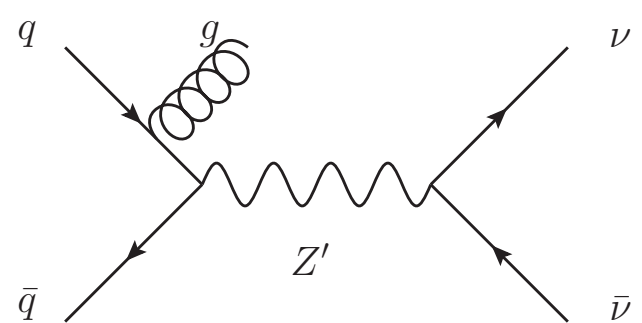

(a) mono-jet: $q \bar{q} \rightarrow g \nu \bar{\nu}$

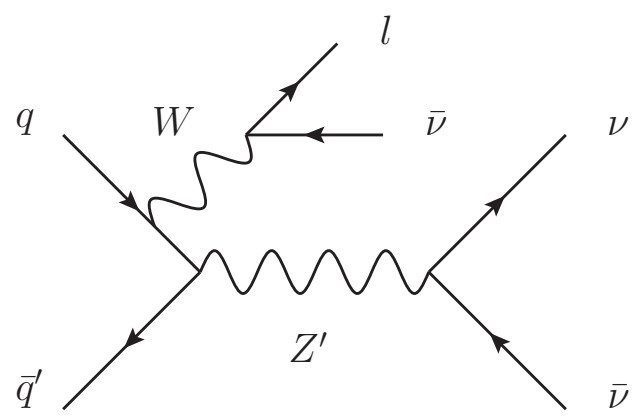

(c) mono-lepton: $q \bar{q}^{\prime} \rightarrow l \bar{\nu} \nu \bar{\nu}$

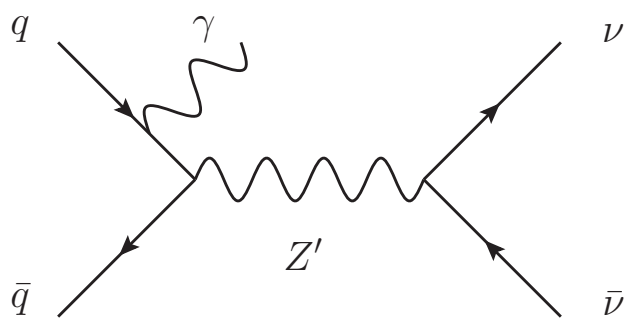

(b) mono-photon: $q \bar{q} \rightarrow \gamma \nu \bar{\nu}$

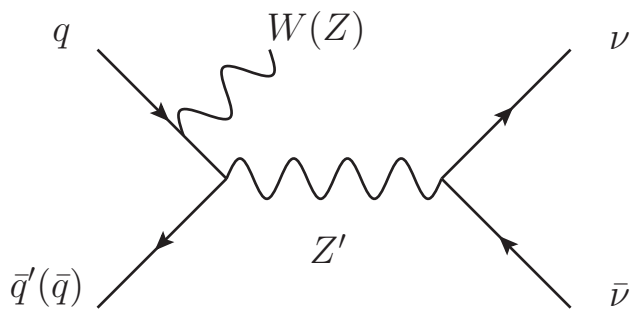

(d) mono- $\mathrm{W}(\mathrm{Z}): q \bar{q}^{\prime}(\bar{q}) \rightarrow W(Z) \nu \bar{\nu}$

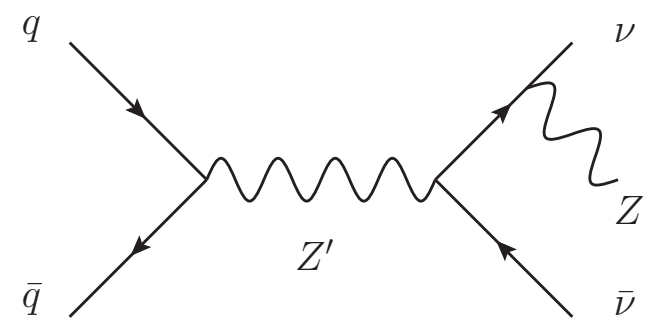

(e) mono-Z: $q \bar{q} \rightarrow Z \nu \bar{\nu}$

Figure 1. Representative Feynman diagrams at leading order for the process $q \bar{q} \rightarrow g \nu \bar{\nu}, q \bar{q} \rightarrow$ $\gamma \nu \bar{\nu}, q \bar{q}^{\prime} \rightarrow l \bar{\nu} \nu \bar{\nu}, q \bar{q}^{\prime}(\bar{q}) \rightarrow W(Z) \nu \bar{\nu}$ and $q \bar{q} \rightarrow Z \nu \bar{\nu}$. Flavor indices of neutrinos are suppressed for simplicity.

For MC simulation of the NSI signals we use a model file generated with FeynRule [93] similar to that used for dark matters with spin-1 mediator [73]. We generate signal samples with MG5_aMC@NLO [94] followed by parton showering and hadronizations with PYTHIA8 [95], and analyse the events with MadAnalysis5 [96]. We use CTEQ6M [97] PDFs in the simulation and use the default renormalization and factorization scale choices in MadGraph5, which is the sum of transverse energy of all final states divided by two. In this section we report results using leading-order calculations matched with parton showering and hadronization. We will discuss the impact of next-to-leading order (NLO) QCD corrections and theoretical uncertainties due to scale variation and choice of parton distribution functions later. 


\subsection{Data selection}

We summarize the LHC data sets used in our study. They include recent measurements of mono-jet [98, 99], mono-V [99, 100], mono-photon [101, 102], and mono-lepton [103, 104] production from both ATLAS and CMS collaborations at LHC $13 \mathrm{TeV}$. The experimental analyses unfold the raw data to particle level with minimal selection cuts. The final measurements are presented in a model-independent form of upper limit on total cross section in different fiducial regions. That ensures a direct comparison to various new physics beyond the standard model which generates large missing transverse momentums. We reproduce the major selection cuts used in all analyses as below for completeness. In the following jets are clustered with anti- $k_{T}$ jet algorithm [105] and a distance parameter of $D=0.4$ unless specified.

We start with measurements on hadronic final states recoiling against large missing energies. The mono-jet production has the largest rate among all processes considered. In the ATLAS analysis it requires a lower threshold on the missing transverse momentum of $p_{T}^{\text {miss }}>250 \mathrm{GeV}$. For the visible objects it requires a leading jet with $p_{T}>250 \mathrm{GeV}$ and $|\eta|<2.4$, and a maximum of four jets with $p_{T}>30 \mathrm{GeV}$ and $|\eta|<2.8$. Furthermore, the separation of each jet and the missing transverse momentum in azimuthal plane should satisfy $\Delta \phi\left(j, \vec{p}_{T}^{\text {miss }}\right)>0.4$. The CMS analysis imposes the same lower threshold of $p_{T}^{\text {miss }}>250 \mathrm{GeV}$, and requires a leading jet with $p_{T}>100 \mathrm{GeV}$ and $|\eta|<2.4$. The separation in azimuthal plane are $\Delta \phi\left(j, \vec{p}_{T}^{\text {miss }}\right)>0.5$ for each of the first four leading jets with $p_{T}>30 \mathrm{GeV}$. Unlike the ATLAS case no jet veto are applied in the CMS analysis. For the production of large missing energies with a single $W / Z$ boson, and subsequent hadronic decays, both ATLAS and CMS collaborations use sophisticated technique of jet substructures to isolate the hadronic decaying $W / Z$ bosons from backgrounds of QCD jets production. However, efficiencies of those selections are derived for specific models, and can be applied to deduce limits on cross sections of production of $W / Z$ boson without decays. In this sense the minimum requirements are a lower threshold of $250 \mathrm{GeV}$ for both the missing transverse momentum and the transverse momentum of the $W / Z$ boson. The ATLAS analysis presents results for final state with $W$ and $Z$ boson separately while CMS analysis only shows results with $W$ and $Z$ boson combined.

In case of production of large missing energies with a $W$ boson, and subsequent leptonic decays, that leads to the mono-lepton signatures. Indeed such final states are indistinguishable from those induced by production of a heavy $W^{\prime}$ boson followed with leptonic decays. The principal variable used in both ATLAS and CMS analyses concerning mono-lepton signature is the transverse mass of the charged lepton and the missing transverse momentum, $m_{T}$. The ATLAS analysis requires electron (muon) candidates to have $|\eta|<1.37$ or $1.52<|\eta|<2.47(|\eta|<2.5)$ and $p_{T}>65(55) \mathrm{GeV}$. The lower threshold on missing transverse momentum $p_{T}^{\text {miss }}$ and the transverse mass $m_{T}$ are $65 \mathrm{GeV}$ and $130 \mathrm{GeV}$ respectively for electron final state, and $55 \mathrm{GeV}$ and $110 \mathrm{GeV}$ for muon. The CMS analysis requires electron (muon) candidates to have $|\eta|<1.44$ or $1.56<|\eta|<2.47(|\eta|<2.4)$ and $p_{T}>130(53)$ $\mathrm{GeV}$. A lower limit of $150 \mathrm{GeV}$ on missing transverse momentum is imposed. In the ATLAS mono-photon analysis it requires a leading photon with $|\eta|<1.37$ or $1.52<|\eta|<2.37$ and 


\begin{tabular}{|c|cccc|}
\hline Label & Variable & Range & Lum. $\left(\mathrm{fb}^{-1}\right)$ & Ranking \\
\hline $\mathrm{CMS}_{J}[99]$ & $p_{T}^{\text {miss }}$ & $1250 \mathrm{GeV}$ & 35.9 & 1 \\
$\mathrm{CMS}_{W+Z}[99]$ & $p_{T}^{\text {miss }}$ & $750 \mathrm{GeV}$ & 35.9 & 2 \\
$\mathrm{ATLAS}_{J}[98]$ & $p_{T}^{\text {miss }}$ & $1000 \mathrm{GeV}$ & 36.1 & 3 \\
$\mathrm{ATLAS}_{Z}[100]$ & $p_{T}^{\text {miss }}$ & $1500 \mathrm{GeV}$ & 36.1 & 4 \\
$\mathrm{CMS}_{\gamma}[102]$ & $p_{T}^{\gamma}$ & $1000 \mathrm{GeV}$ & 35.9 & 5 \\
$\mathrm{ATLAS}_{\gamma}[101]$ & $p_{T}^{\gamma}$ & $1000 \mathrm{GeV}$ & 36.1 & 6 \\
$\mathrm{ATLAS}_{W}[100]$ & $p_{T}^{\text {miss }}$ & $1500 \mathrm{GeV}$ & 36.1 & 7 \\
$\mathrm{ATLAS}_{e}[104]$ & $m_{T}^{\text {min }}$ & $5127 \mathrm{GeV}$ & 139 & 8 \\
$\mathrm{ATLAS}_{\mu}[104]$ & $m_{T}^{\text {min }}$ & $5127 \mathrm{GeV}$ & 139 & 9 \\
$\mathrm{CMS}_{e}[103]$ & $m_{T}^{\text {min }}$ & $5127 \mathrm{GeV}$ & 35.9 & 10 \\
$\mathrm{CMS}_{\mu}[103]$ & $m_{T}^{\text {min }}$ & $5127 \mathrm{GeV}$ & 35.9 & 11 \\
\hline
\end{tabular}

Table 1. Summary of various information on data sets used in this study, including the principal variable used in each data set, its highest value probed, the total luminosity, and a ranking of different data sets.

$p_{T}^{\gamma}>150 \mathrm{GeV}$, and $\Delta \phi\left(\gamma, p_{T}^{\text {miss }}\right)>0.4$. The CMS analysis requires a leading photon with $p_{T}^{\gamma}>175 \mathrm{GeV}$ and $|\eta|<1.44$. In addition the missing transverse momentum should satisfy $p_{T}^{\text {miss }}>170 \mathrm{GeV}$ and $p_{T}^{\gamma} / p_{T}^{\text {miss }}<1.4$.

In all above analyses the measured cross sections are binned in the principal variables, which are $p_{T}^{\text {miss }}, p_{T}^{\gamma}$, and $m_{T}$ for mono-jet and mono- $V$, mono-photon, and mono-lepton, respectively. Each bin in the principal variable is also called an exclusive region. Besides, ATLAS and CMS also measure the cumulated cross sections from a lower threshold of the principal variable to almost the largest value allowed. Each of those selected kinematic range is called an inclusive region. We use the cross sections measured in inclusive regions to constrain the non-standard interactions in our analysis by default, and compare results to those obtained from exclusive regions if the latter is available. In table. 1 we summarize further information on the LHC data sets used in our analysis. That includes the total luminosity corresponds to each measurement, the largest sensible values of the principal variable probed in each measurement, and a ranking on different measurements according to the constraint derived. The CMS mono-jet measurement sets the strongest constraint on the non-standard interactions, followed by the CMS mono- $W / Z$ measurement, ATLAS mono-jet and mono- $Z$ measurements.

We explain briefly the statistical procedure used to derive exclusion limit on the nonstandard interactions. We use the CLs [106] method together with the log-likelihood $\chi^{2}$ as a function of the model parameters and the signal strength $\mu$,

$$
\chi^{2}\left(\mu, \epsilon, M_{Z^{\prime}}\right)=\sum_{i=1}^{n} \frac{\left(N_{\mathrm{obs}, i}-N_{b g, i}-\mu \sigma_{i}\left(\epsilon, M_{Z^{\prime}}\right) \mathcal{L}\right)^{2}}{N_{\mathrm{obs}, i}+\delta_{\text {sys }, i}^{2}}=\chi_{0}^{2}+A \mu+B \mu^{2},
$$

for each data set and with $i$ runs from all regions considered. For each region, $N_{o b s, i}$ and 
$N_{b g, i}$ are the total number of events observed and predicted by the SM, $\delta_{s y s, i}$ is the total systematic error, and $\sigma_{i}\left(\epsilon, M_{Z^{\prime}}\right)$ represents the cross section predicted by the model of non-standard interactions. $\mathcal{L}$ is the integrated luminosity. The $\chi^{2}$ is a quadratic function of $\mu$ with coefficients $A, B$ and $\chi_{0}^{2}$ depending on model parameters $M_{Z^{\prime}}$ and $\epsilon$. CLs upper limit on the NSI strength $\epsilon$ for fixed $M_{Z^{\prime}}$ at a confidence level 1- $\alpha^{\prime}$ is determined by

$$
\hat{\mu}+\Delta_{\mu} \Phi^{-1}\left(1-\alpha^{\prime} \Phi\left(\hat{\mu} / \Delta_{\mu}\right)\right)=1,
$$

with $\hat{\mu}=-A / 2 B, \Delta_{\mu}=1 / \sqrt{B}$. $\Phi$ is the cumulative distribution function of normal distribution. In case of using exclusive region/bin we can include all regions of the data set into $\chi^{2}$ to derive the limit on $\epsilon$ if bin-bin experimental correlations are known. For using inclusive region, we can only include one of them at a time since different inclusive regions are statistically correlated. For a single inclusive region/bin, the CLs limit on the cross section induced by non-standard interactions can be simplified as

$$
\sigma_{\text {up }}=\hat{\sigma}+\Delta_{\sigma} \Phi^{-1}\left(1-\alpha^{\prime} \Phi\left(\hat{\sigma} / \Delta_{\sigma}\right)\right)
$$

with the maximum likelihood estimator and the uncertainty of $\sigma$ given by

$$
\hat{\sigma}=\left(N_{\text {obs }}-N_{\text {bg }}\right) / \mathcal{L}, \quad \Delta_{\sigma}=\sqrt{N_{\text {obs }}+\delta_{\text {sys }}^{2}} / \mathcal{L} .
$$

In our analysis for each data set we scan over all the inclusive regions for the exclusive limit on $\epsilon$ and take the strongest one among them. We have verified explicitly with the CMS mono-jet measurement that the exclusion limit as derived from a scan on the inclusive regions is similar to that obtained using a $\chi^{2}$ with all exclusive regions.

\subsection{Constraints for effective operator}

We first present results for case of using effective operator. In figure 2 we show the contour of 95\% CLs upper limit on the plane of the NSI $\epsilon_{u}$ and $\epsilon_{d}$, from all LHC data sets discussed earlier. We only include one representative result for mono-lepton from CMS for simplicity. The effective parton-parton center-of-mass energy is approximately $5 \mathrm{TeV}$ for $13 \mathrm{TeV}$ run of LHC. It indicates the new physics scale $\Lambda$ in the effective operator approach should be larger than that to ensure its validity. Meanwhile, the Wilson coefficient $c$ in eq. (2.2) can not exceed a perturbative bound of $4 \pi$ assuming it is induced by tree-level amplitude in a weakly coupled theory. That sets a boundary value of about 0.015 for the NSIs as shown by the dashed horizontal and vertical lines in figure 2. Outside the bounded region the effective operator approach is not valid at the LHC if one does not apply any cut on the center-of-mass energy of the scattering. Furthermore, for NSIs with left-handed quarks, to maintain the gauge invariance of SM SU $(2)_{L}$ symmetry one should set $\epsilon_{u}=\epsilon_{d}$. Otherwise it may lead to apparently too strong constraints on the NSIs in the direction of $\epsilon_{u}=-\epsilon_{d}$ due to violation of gauge invariance [80], as can be seen for mono- $W$ and mono-lepton production in figure 2. In the following we will focus on constraints along diagonal direction $\epsilon_{u}=\epsilon_{d}=\epsilon$.

The CMS mono-jet measurement sets the strongest constraint of $\epsilon \lesssim 0.011$, followed by CMS mono- $W / Z$ with constraints of $\epsilon \lesssim 0.015$, both within the perturbative region, and 


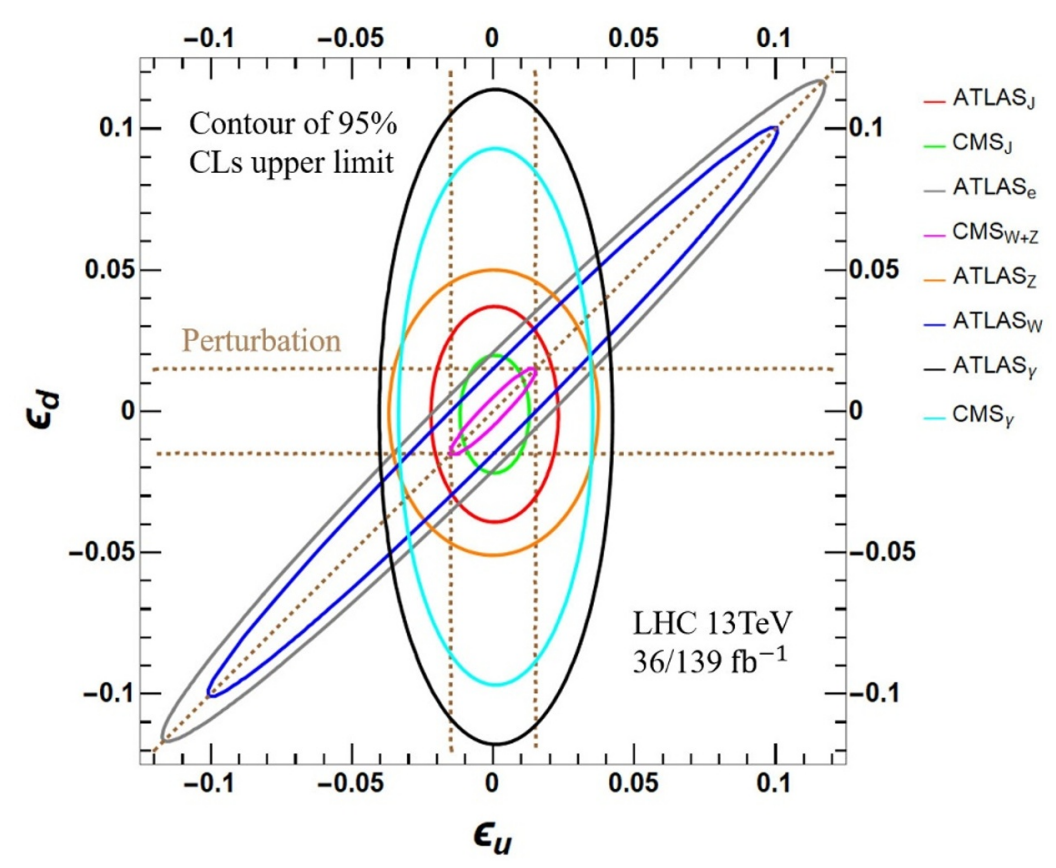

Figure 2. Contour of 95\% CLs upper limit on the plane of NSIs $\epsilon_{u}$ and $\epsilon_{d}$ in the framework of effective operators with various measurements at the LHC. The vertical and horizontal dashed lines indicate bounds from perturbative conditions.

ATLAS mono-jet with constraints of $\epsilon \lesssim 0.020$. Measurements on mono-photon and monolepton production lead to much weaker constraints. The constraints are almost symmetric in the positive and negative directions due to the relatively smallness of interference effects with SM for the NSI strength probed. The asymmetry can be measured by the difference of $\epsilon^{+}$and $\epsilon^{-}$, which are bounds in the positive and negative directions respectively. For the case of CMS mono-jet production, we have $\left(\left|\epsilon^{+}\right|-\left|\epsilon^{-}\right|\right) /\left|\epsilon^{+}\right| \sim 2 \%$. It is interesting that CMS measurements in general impose stronger constraints than ATLAS for the same final states due to the smaller systematic uncertainties of CMS.

\subsection{Constraints for simplified $Z^{\prime}$ model}

We turn to the constraints for NSIs from simplified $Z^{\prime}$ model. The production cross sections at the LHC depend on couplings of the $Z^{\prime}$ boson to quarks, neutrinos, as well as on mass and width of the boson, $M_{Z^{\prime}}$ and $\Gamma_{Z^{\prime}}$. Similar as before we would like to translate the constraints to the conventional NSI parameter $\epsilon_{u}=\epsilon_{d}=\epsilon$ defined in eq. (2.4). For a fixed value of $\epsilon$, one can still vary $M_{Z^{\prime}}$ and $\Gamma_{Z^{\prime}}$ for changes of cross sections at the LHC. We fix $\Gamma_{Z^{\prime}} / M_{Z^{\prime}}=0.1$ and study the constraints on $\epsilon$ as a function of the mass of $Z^{\prime}$ boson for simplicity. In figure 3 we summarize the constraints imposed by mono-jet and mono- $V$ measurements from both ATLAS and CMS. We show the 95\% CLs upper limits on the positive axis of $\epsilon$. The limits on negative side of the axis are quite similar since the interference effects are small in general. For example, in CMS mono-jet production with 


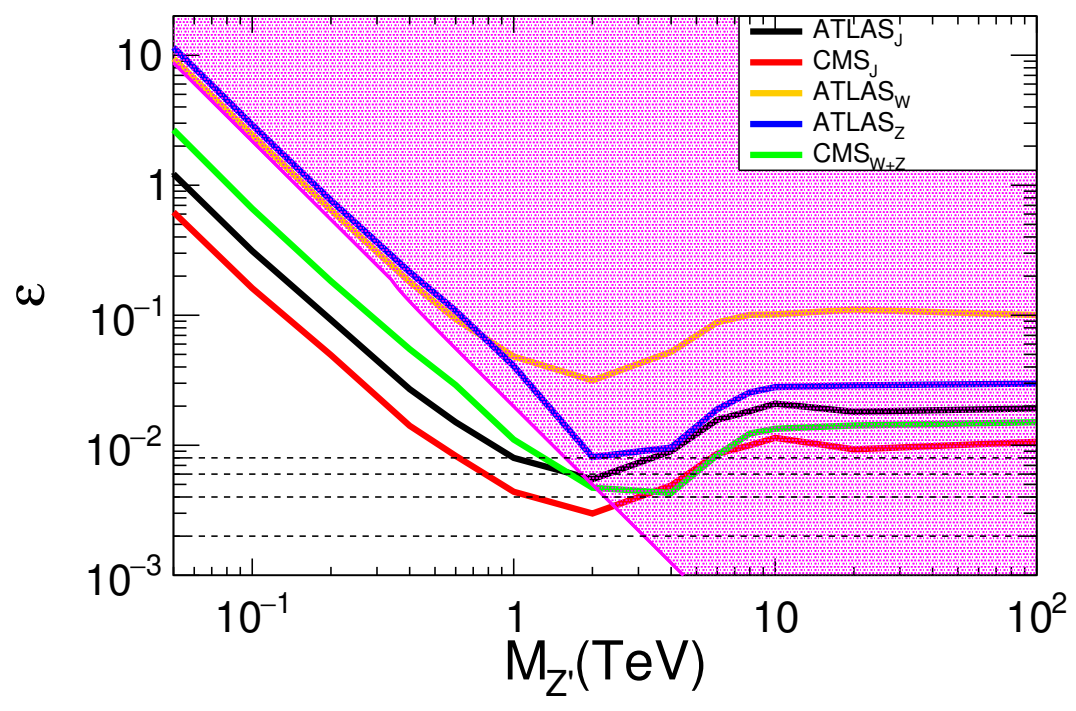

Figure 3. 95\% CLs upper limit on NSIs in a simplified $Z^{\prime}$ model as a function of the mass of $Z^{\prime}$ with various measurements at the LHC. We assume $\epsilon_{u}=\epsilon_{d}=\epsilon$ and $\Gamma_{Z^{\prime}} / M_{Z^{\prime}}=0.1$.
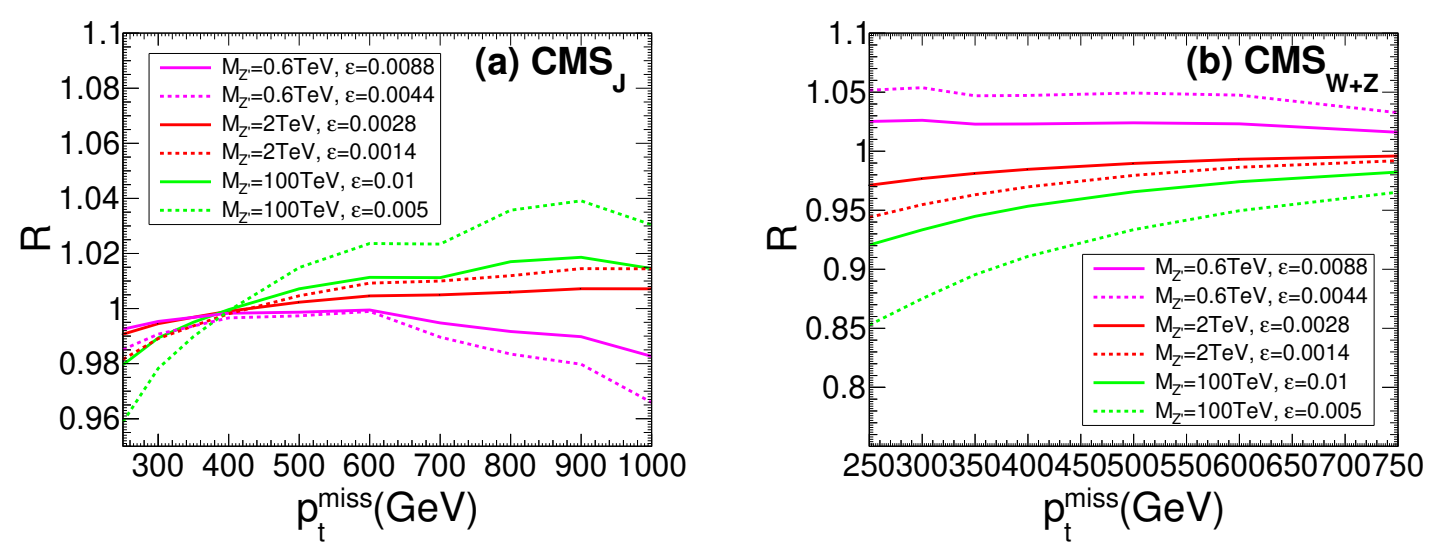

Figure 4. Ratio of predicted cross-sections without and with interference contributions, for production of CMS mono-jet and mono- $W / Z$ on the left and right respectively. Results are shown for a few benchmark points of $M_{Z^{\prime}}$ and $\epsilon$ according to current and future LHC sensitivity.

$M_{Z^{\prime}}=2 \mathrm{TeV}$, the value of $\left(\left|\epsilon^{+}\right|-\left|\epsilon^{-}\right|\right) /\left|\epsilon^{+}\right|$is about $0.5 \%$. The impact of interference between NSIs and SM can be further visualized in figure 4, where we show ratios between predicted cross-sections without and with including interference contributions. We choose a few benchmark points of $M_{Z^{\prime}}$ and $\epsilon$ that are close to the current and future sensitivity of LHC. We can see for the large transverse momentum region, which has the strongest sensitivity to NSIs, the interference terms contribute less than $10 \%$ to the cross sections. For a certain choice of $M_{Z^{\prime}}$ the NSI $\epsilon$ can not be arbitrarily large otherwise the partial widths of $Z^{\prime}$ decaying into neutrinos and quarks can easily saturate the assumed total width. That 
leads to a theoretical upper bound on the NSI as [62]

$$
|\epsilon| \leq \frac{\sqrt{3} \pi}{\sqrt{N} G_{F} M_{Z^{\prime}}^{2}} \frac{\Gamma_{Z^{\prime}}}{M_{Z^{\prime}}},
$$

where $N$ is the number of massless quarks plus possible contributions from heavy quarks with mass below $M_{Z^{\prime}} / 2$. The parameter space in figure 3 with colors are thus excluded.

The CMS measurement on mono-jet production again gives the strongest constraints, with an upper limit ranging from about 0.6 for a $Z^{\prime}$ mass of $50 \mathrm{GeV}$ to 0.0028 for a $Z^{\prime}$ mass of $2 \mathrm{TeV}$. The constraints become weaker when $M_{Z^{\prime}}$ goes beyond $2 \mathrm{TeV}$ since then the $Z^{\prime}$ boson can hardly be produced directly. The ATLAS mono-jet measurement sets a limit of about two times larger than CMS. Our results on constraints from ATLAS mono-jet production agree well with that shown in ref. [62]. The constraints from CMS mono- $V$ measurement are weaker than those from ATLAS mono-jet except for very large $M_{Z^{\prime}}$. The constraints from ATLAS mono- $Z / W$ measurements are weaker than the theoretical bounds. Interestingly, results for the $Z^{\prime}$ model approach smoothly to those of effective operators with increasing $Z^{\prime}$ mass, as demonstrated in figure 3 . In all cases the limits are strongest for a $Z^{\prime}$ mass of about $2 \mathrm{TeV}$, and increase afterwards, and finally stabilize for $M_{Z^{\prime}} \gtrsim 6 \mathrm{TeV}$. Results shown in figure 3 can also be translated into constraints for different choices of $\Gamma_{Z^{\prime}} / M_{Z^{\prime}}$ easily. For instance, if instead assuming $\Gamma_{Z^{\prime}} / M_{Z^{\prime}}=5 \%$, constraints from all data sets will scale down by $1 / \sqrt{2}$ since the cross sections are approximately proportional to $\epsilon^{2} / \Gamma_{Z^{\prime}}$ for not too heavy $Z^{\prime}$. Meanwhile, the theoretical bounds will scale down by a factor of 2 and are more closer to the experimental constraints.

\section{Theoretical uncertainties}

In this section we extend our results by using theoretical predictions calculated at next-toleading order in QCD. The NLO QCD corrections can be potentially large in tail region of various distributions, that have the strongest sensitivity to NSIs. We further study impact of theoretical uncertainties on the constraints to NSIs, including the scale variations and uncertainties due to parton distribution functions.

\subsection{Next-to-leading order QCD corrections}

The NLO calculations for various processes mentioned earlier can be carried out straightforwardly by generating the model file of NSIs at NLO in QCD with FeynRules [93] followed by simulation with MG5_aMC@NLO [94] and PYTHIA8 [95]. The impact of corrections to various distributions can be described by a K-factor defined as

$$
K\left(O_{0}\right)=\frac{\sigma_{N L O}\left(O>O_{0}\right)}{\sigma_{L O}\left(O>O_{0}\right)}
$$

calculated for different inclusive regions, where the numerator and denominator are cumulated cross sections at NLO and LO respectively. Our nominal predictions are calculated with the default choice of QCD renormalization and factorization scales, and with the central set of CTEQ6M NLO PDFs [97]. We vary the renormalization and factorization scales 

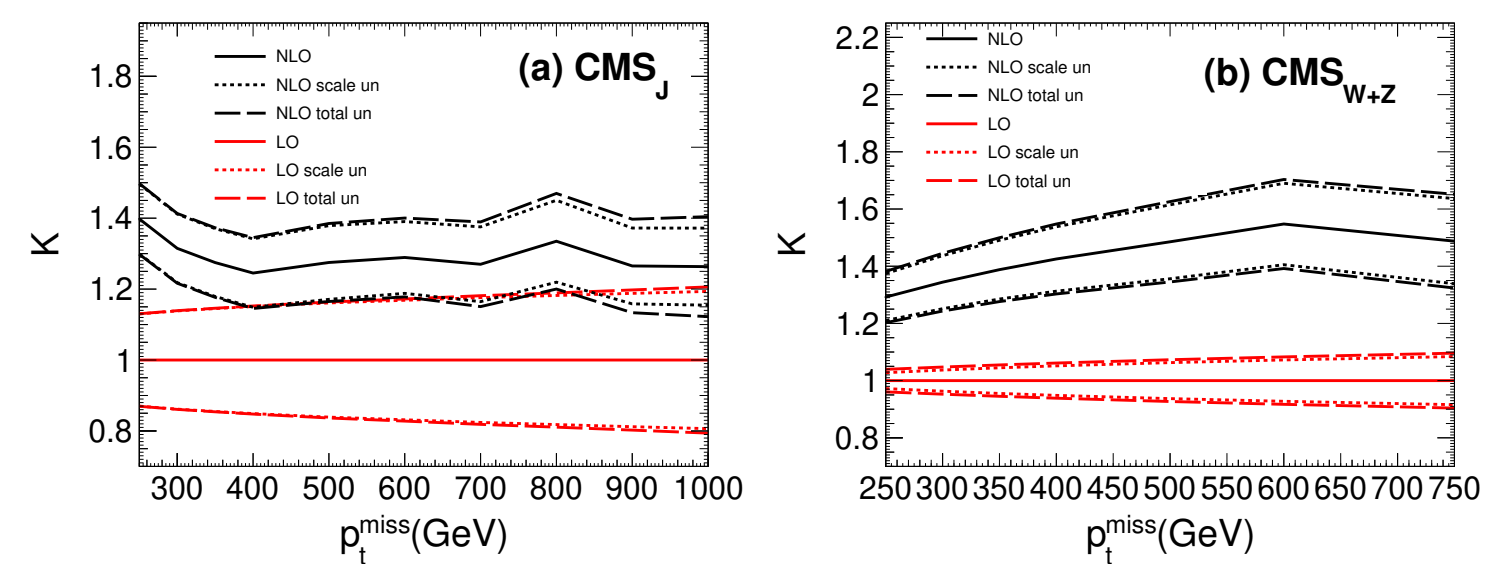

Figure 5. K-factors defined as ratio of NLO cross sections to LO cross sections for inclusive regions as a function of the lower threshold for CMS mono-jet and mono- $W / Z$ on the left and right respectively, for a $Z^{\prime}$ mass of $100 \mathrm{GeV}$. The dashed (dotted) lines show the total (scale) uncertainties of the LO and NLO predictions.

independently by a factor of two and take the 9-scale envelope as the uncertainty range. The PDF uncertainties are calculated with Hessian error sets provided in CTEQ6M PDFs at $68 \%$ C.L. The total theoretical uncertainties are quadratic sum of the scale and PDF uncertainties in both plus and minus directions.

In figures 5 and 6 we plot the K-factor as a function of the lower threshold of principal observable for CMS mono-jet production and CMS mono- $W / Z$ production, with the $Z^{\prime}$ mass of $100 \mathrm{GeV}$ and $1 \mathrm{TeV}$ respectively. NLO corrections and theoretical uncertainties are quite similar for the case of ATLAS mono-jet and mono- $V$ which we do not show for simplicity. The solid line represents the K-factor and the dashed (dotted) lines show the total (scale) uncertainty for LO and NLO predictions. The QCD corrections start from 40 (45)\% at low $p_{T}^{\text {miss }}$ and decrease to about 25 (10)\% for mono-jet production with $M_{Z^{\prime}}=100 \mathrm{GeV}$ $(1 \mathrm{TeV})$. For mono- $W / Z$ production the QCD corrections are about $30(20) \%$ at low $p_{T}^{\text {miss }}$ and increase to about $50(25) \%$ slowly with $M_{Z^{\prime}}=100 \mathrm{GeV}(1 \mathrm{TeV})$. The peculiar shape of $\mathrm{K}$-factor in mono-jet plot with $M_{Z^{\prime}}=100 \mathrm{GeV}$ is partly due to the MC errors. In all cases uncertainties due to scale variations are dominant over PDF uncertainties. We observe a reduction of scale uncertainties for NLO predictions except for mono- $W / Z$ production with $M_{Z^{\prime}}=100 \mathrm{GeV}$ where the scale variations at $\mathrm{LO}$ underestimate the genuine perturbative uncertainties. The total uncertainties increase with $p_{T}^{\text {miss }}$ at both LO and NLO. For NLO predictions the relative total uncertainties range between $7 \sim 11 \%$ for mono-jet production with two choices of masses, and between $4 \sim 10 \%$ for mono- $W / Z$ production.

\subsection{Constraints at NLO}

We are now ready to study impact of the NLO corrections and theoretical uncertainties on the derived limit of NSIs. The results are presented in figure 7 as a function of the mass of $Z^{\prime}$ for constraint with CMS mono-jet and mono- $W / Z$ production respectively. We derive four groups of $95 \%$ CLs upper limit on $\epsilon$. They include the two using our nominal LO and 

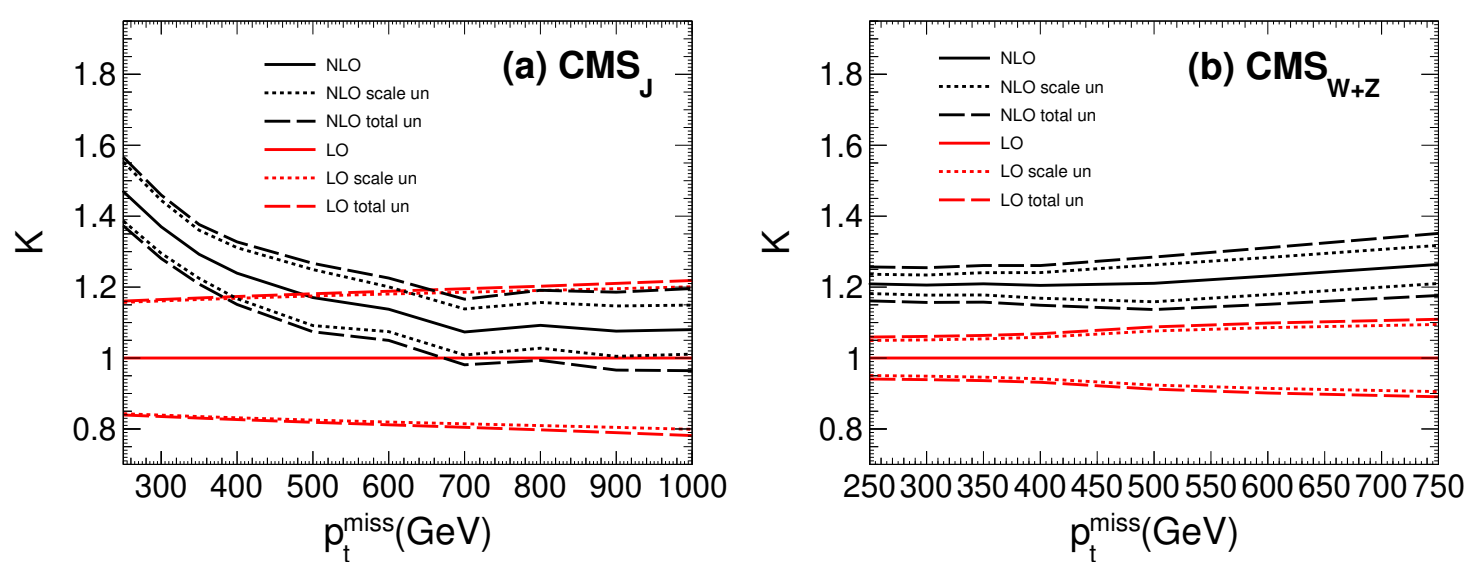

Figure 6. K-factors defined as ratio of NLO cross sections to LO cross sections for inclusive regions as a function of the lower threshold for CMS mono-jet and mono- $W / Z$ on the left and right respectively, for a $Z^{\prime}$ mass of $1 \mathrm{TeV}$. The dashed (dotted) lines show the total (scale) uncertainties of the LO and NLO predictions.

NLO predictions on the cross sections. In the other two we use the LO predictions scaled to the lower side of the uncertainty band and similar for NLO predictions, which corresponds to conservative constraints on NSIs than those using nominal theory predictions. We plot all four constraints normalized to the one with nominal LO predictions as a function of $M_{Z^{\prime}}$ in figure 7 .

For the case of CMS mono-jet production, the theoretical uncertainties weaken the limit by $10 \sim 15 \%$ at LO across the full range of $M_{Z^{\prime}}$. The NLO corrections increase the cross sections and thus lead to an improvement of $5 \sim 10 \%$ on the constraints of NSIs. The theoretical uncertainties have less impact at NLO than at LO due to the stabilization of theory predictions at higher orders. In combination with NLO corrections and theory uncertainties the constraints on NSIs improve slightly as comparing to the nominal LO ones that we show in section 2. For CMS mono- $W / Z$ production, the theoretical uncertainties change the limit by less than $10 \%$ at LO and even smaller at NLO. The constraints on NSIs are improved by $10 \%$ in the full range of $M_{Z^{\prime}}$ when considering the NLO corrections together with theoretical uncertainties.

\section{LHC combination and projections}

We have shown that for individual measurement the strongest constraints on NSIs arise from CMS mono-jet production in both the EFT framework and the simplified $Z^{\prime}$ model. It is worth to study the improvement once we combine constraints from several data sets, specifically the CMS mono-jet, CMS mono- $W / Z$, and ATLAS mono-jet measurements. For each value of the $Z^{\prime}$ mass, we first identify the most sensitive inclusive region for each of the three measurements. We construct the total $\chi^{2}$ function in eq. (2.5) as a sum of the three individual $\chi^{2}$. The $95 \%$ CLs upper limit is then determined following the prescription outlined earlier. We neglect correlations between systematic errors of different 

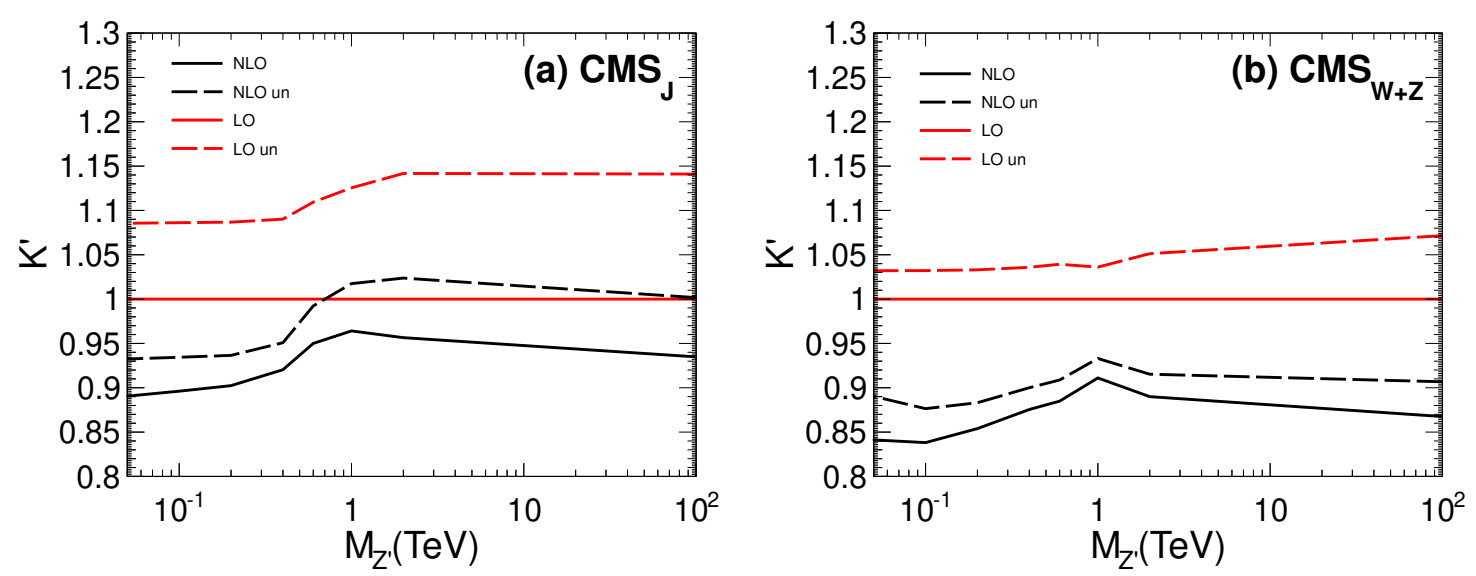

Figure 7. Exclusion limits on NSIs using different theoretical predictions normalized to those using leading order predictions without theoretical uncertainties, as a function of the mass of $Z^{\prime}$. The left (right) plots corresponds to constraints from CMS mono-jet (mono- $W / Z$ ) measurement.

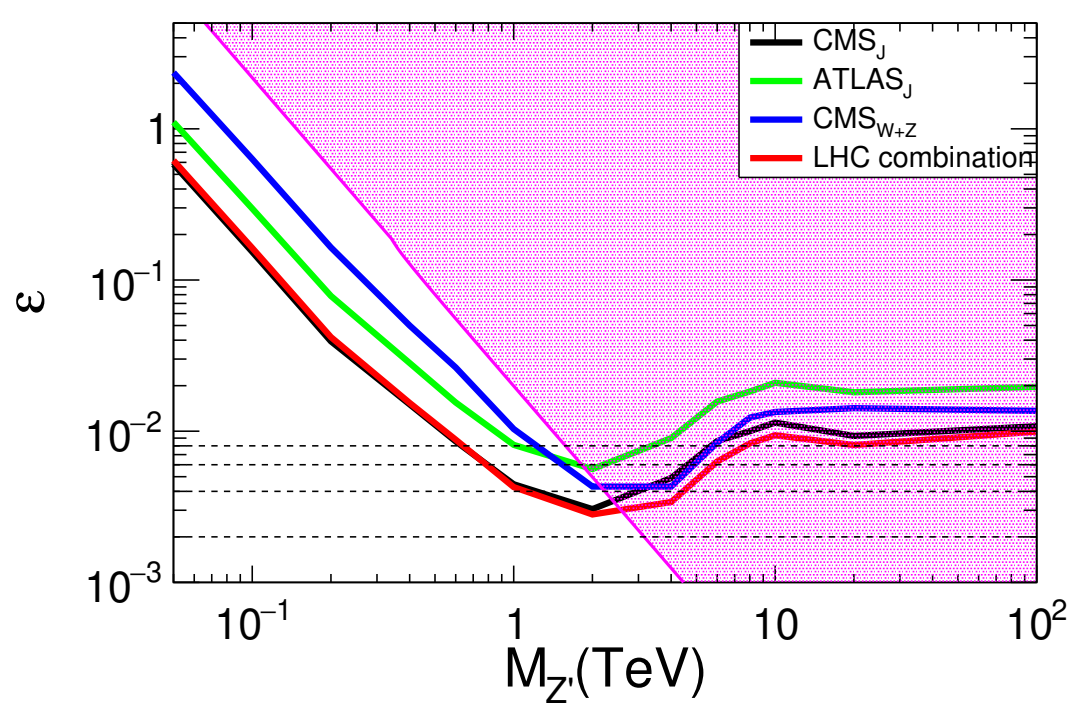

Figure 8. 95\% CLs upper limit on NSIs in a simplified $Z^{\prime}$ model as a function of the mass of $Z^{\prime}$ with various measurements at the LHC and their combinations. We assume $\epsilon_{u}=\epsilon_{d}=\epsilon$ and $\Gamma_{Z^{\prime}} / M_{Z^{\prime}}=0.1$, and use the NLO predictions with theoretical uncertainties.

measurements which are not available. The results are presented in figure 8 using the NLO predictions with theoretical uncertainties. For $M_{Z^{\prime}}$ below $1 \mathrm{TeV}$ the combined limits are almost identical to those from CMS mono-jet alone since the latter are stronger by more than a factor of two than other data sets. The constraints are improved by at least about $10 \%$ for $M_{Z^{\prime}}$ greater than $1 \mathrm{TeV}$.

The LHC is expected to accumulate a total integrated luminosity of $3000 \mathrm{fb}^{-1}$ for the high luminosity run. The constraints on NSIs can benefit from the high statistics of various measurements. We calculate the projections for constraints on NSIs with mono-jet 


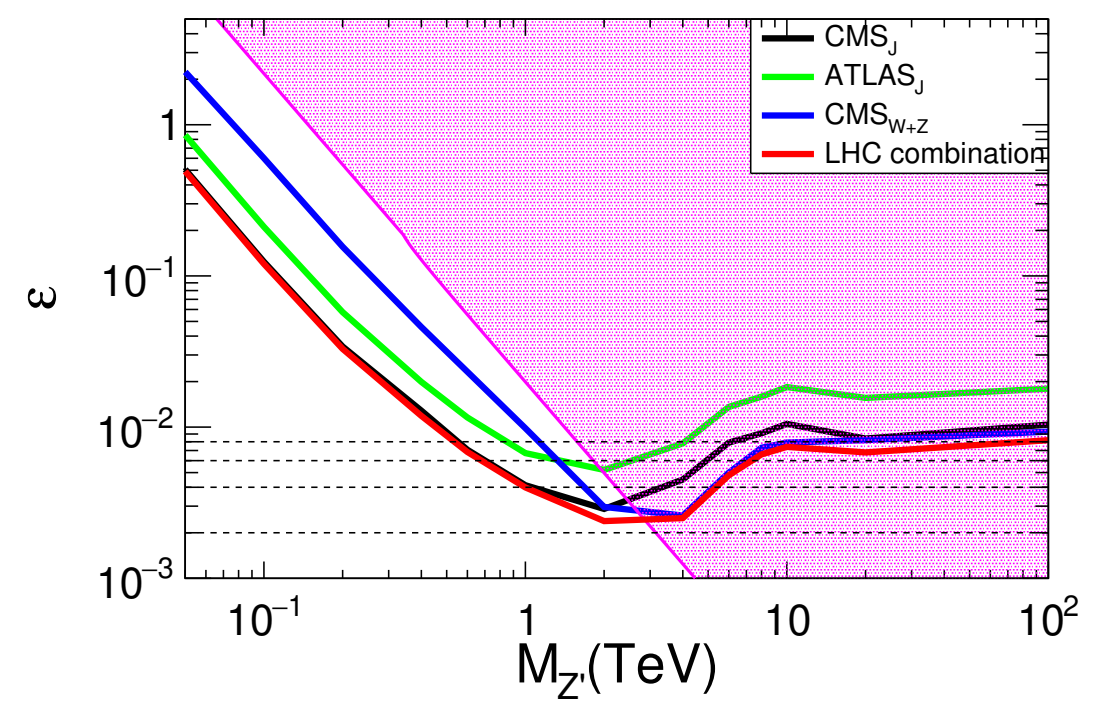

Figure 9. Projection of exclusion limit on NSIs for LHC with an integrated luminosity of $300 \mathrm{fb}^{-1}$, in a simplified $Z^{\prime}$ model as a function of the mass of $Z^{\prime}$. We assume $\epsilon_{u}=\epsilon_{d}=\epsilon$ and $\Gamma_{Z^{\prime}} / M_{Z^{\prime}}=0.1$, and use the NLO predictions with theoretical uncertainties.

and mono- $W / Z$ measurements at LHC with higher luminosities. We rescale the number of SM background events from current values with luminosities and set the number of observed events to be the same as the SM backgrounds. We assume the relative size of systematic uncertainties remain the same though one may expect certain improvements from both experimental and theoretical sides. In figure 9 we plot the expected upper limit on the NSIs as a function of $M_{Z^{\prime}}$ for an integrated luminosity of $300 \mathrm{fb}^{-1}$. The increase of statistics improves the constraints as from CMS mono- $W / Z$ production while has less impact on mono-jet production since the measurements are already dominated by systematic uncertainties. It is interesting to find the constraints from CMS mono- $W / Z$ measurement become as good as those from CMS mono-jet measurement for $M_{Z^{\prime}}>2 \mathrm{TeV}$.

We summarize the limits on $\epsilon$ in table. 2 for several choices of the mass of $Z^{\prime}$ and different measurements. It is understood that the case with $M_{Z^{\prime}}=100 \mathrm{TeV}$ is equivalent to that using the EFT approach. The current best limit is $\epsilon \lesssim 0.0028$ for $M_{Z^{\prime}}=2 \mathrm{TeV}$ with combination of the three measurements. We expect reducing the limit to 0.0025 with $300 \mathrm{fb}^{-1}$ data at the LHC. We note that all limits presented so far are for the choice of $Z^{\prime}$ width $\Gamma_{Z^{\prime}} / M_{Z^{\prime}} \equiv r=0.1$. As mentioned earlier the constraints on $\epsilon$ scale as $\sqrt{r}$ approximately. Thus for $r=0.05$ the current best limit would be $\epsilon \lesssim 0.0020$. We also calculate the projections for HL-LHC with a luminosity of $3000 \mathrm{fb}^{-1}$, and only find improvements of a few percents for the limits on NSIs in the full range of $M_{Z^{\prime}}$ considered. However, in future measurements with high statistics, one can further extend the measured $p_{T}^{\text {miss }}$ to even higher values. That requires a dedicated study on the SM backgrounds in that region, and we expect more improvements can be gained than those shown in table. 2 . 


\begin{tabular}{|c|ccc|cccc|ccc|}
\hline & \multicolumn{3}{|c}{ Current data } & \multicolumn{3}{c|}{ HL-LHC $\left(300 \mathrm{fb}^{-1}\right)$} & \multicolumn{3}{c|}{ HL-LHC $\left(3000 \mathrm{fb}^{-1}\right)$} \\
\hline$M_{Z^{\prime}}(\mathrm{TeV})$ & 0.2 & 2 & 100 & 0.2 & 2 & 100 & 0.2 & 2 & 100 \\
\hline $\mathrm{CMS}_{J}$ & 0.039 & 0.0031 & 0.011 & 0.035 & 0.0030 & 0.010 & 0.035 & 0.0028 & 0.0097 \\
$\mathrm{CMS}_{W+Z}$ & 0.16 & 0.0043 & 0.014 & 0.16 & 0.0030 & 0.0093 & 0.15 & 0.0028 & 0.0088 \\
ATLAS $_{J}$ & 0.078 & 0.0056 & 0.020 & 0.057 & 0.0052 & 0.018 & 0.057 & 0.0052 & 0.018 \\
Combined & 0.042 & 0.0028 & 0.010 & 0.035 & 0.0025 & 0.0081 & 0.033 & 0.0023 & 0.0077 \\
\hline
\end{tabular}

Table 2. Summary of current and projected $95 \%$ CLs upper limit on NSIs in a simplified $Z^{\prime}$ model with $Z^{\prime}$ mass of $0.2,2$, and $100 \mathrm{TeV}$ respectively. We assume $\epsilon_{u}=\epsilon_{d}=\epsilon$ and $\Gamma_{Z^{\prime}} / M_{Z^{\prime}}=0.1$, and use the NLO predictions with theoretical uncertainties.

LHC constraints on NC NSIs based on missing transverse energy have also been studied in previous works $[61,62]$. It is noted that the data set taken by both works are ATLAS mono-jet production with $36.1 \mathrm{fb}^{-1}$, and therefore partly overlapped with this paper. Our results concerning ATLAS mono-jet production are consistent with [62], however, less stringent than [61]. By utilizing the more precise data from CMS mono-jet production with $35.9 \mathrm{fb}^{-1}$ data, we have set a limit stronger by a factor of two than [62], which can be read from table. 2. Projections for future run of LHC with integrated luminosities of $300 \mathrm{fb}^{-1}$ and $3000 \mathrm{fb}^{-1}$ have also been given in previous studies. With consideration of reduced systematic uncertainties, they expect larger improvement than in our study of which rather conservative assumptions are taken. On the other hand, there exist indirect searches of NSIs at the LHC utilizing SM gauge symmetries. Under consideration of the common $\mathrm{U}(1)^{\prime}$ coupling shared by the $\mathrm{SU}(2)_{L}$ doublet, process $p p \rightarrow Z^{\prime} \rightarrow l^{+} l^{-}+X$ is taken into account, and measurements on dilepton final state are used to set limits on $\mathrm{NC}$ NSIs [57]. Due to the better sensitivities for final state with charged leptons, a stringent limit has been obtained on the common coupling $g$ between $Z^{\prime}$ and fermions, to be about $10^{-2}$ for $M_{Z^{\prime}} \approx 1 \mathrm{TeV}$, based on ATLAS searches of dilepton resonances [107]. This limit can be converted into a limit on conventional coupling strength of NSIs, $\epsilon \lesssim 10^{-5}$ through eq. (2.4). Finally, it is worth noting that LHC constraints are fairly loose for light mediators with mass smaller than electro-weak scale. For $M_{Z^{\prime}} \approx 50 \mathrm{MeV}$, a limit of $\epsilon \lesssim 0.1$ has been reached with the COHERENT experiment [57, 108].

\section{Conclusion}

The study on possible non-standard interactions of neutrinos with matter has a long history, and stringent limits have been imposed from various experiments. The NSIs can affect the production, propagation as well as detection of neutrinos, and have a direct consequence on global analysis of neutrino properties like mass ordering and CP phases. The successful operation of LHC opens new opportunities on searching for neutrino NSIs at high momentum transfers which are complementary to other experiments. Neutrinos appear as signal of missing transverse momentums in detectors same as those from dark matters. There have been several studies on constraining NC NSIs using measurements of mono-jet production 
at the LHC [60-62]. In our study we select various data sets from LHC measurements at $13 \mathrm{TeV}$ with integrated luminosities of $35 \sim 139 \mathrm{fb}^{-1}$, including production of a single jet, photon, $W / Z$ boson, or charged lepton, accompanied with large missing transverse momentums. We derive constraints on neutral-current NSIs with quarks imposed by different data sets in a framework of either effective operators or simplified $Z^{\prime}$ models.

We found the CMS measurement on mono-jet production gives the strongest constraints on NSIs followed by the CMS measurement on mono- $W / Z$ production. The ATLAS mono-jet measurement also leads to comparable constraints while the mono-photon and mono-lepton production show less sensitivities. We use theoretical predictions of various production induced by NSIs calculated at next-to-leading in QCD matched with parton showering and hadronizations. The inclusion of higher order QCD effects stabilize the theory predictions and result in more robust constraints. In the framework of effective operators we find a $95 \%$ CLs upper limit of 0.010 on the conventional NSI strength parameter $\epsilon$. In a simplified $Z^{\prime}$ model we obtain an upper limit on $\epsilon$ of 0.042 and 0.0028 for a $Z^{\prime}$ mass of 0.2 and $2 \mathrm{TeV}$ respectively, assuming $\Gamma_{Z^{\prime}} / M_{Z^{\prime}}=0.1$. Moreover, we discuss possible improvements from future runs of LHC with higher luminosities. We find a moderate reduction of the limits if using the same experimental setups but expect further gains by extending current measured missing transverse momentums to higher values.

\section{Acknowledgments}

This work was sponsored by the National Natural Science Foundation of China under the Grant No. 11875189 and No.11835005, and by the MOE Key Lab for Particle Physics, Astrophysics and Cosmology.

Open Access. This article is distributed under the terms of the Creative Commons Attribution License (CC-BY 4.0), which permits any use, distribution and reproduction in any medium, provided the original author(s) and source are credited.

\section{References}

[1] P. Minkowski, $\mu \rightarrow$ er at a Rate of One Out of $10^{9}$ Muon Decays?, Phys. Lett. B 67 (1977) 421 [INSPIRE].

[2] R.N. Mohapatra and G. Senjanović, Neutrino Masses and Mixings in Gauge Models with Spontaneous Parity Violation, Phys. Rev. D 23 (1981) 165 [INSPIRE].

[3] T. Yanagida, Horizontal Symmetry and Masses of Neutrinos, Prog. Theor. Phys. 64 (1980) 1103 [INSPIRE].

[4] R. Foot, H. Lew, X.G. He and G.C. Joshi, Seesaw Neutrino Masses Induced by a Triplet of Leptons, Z. Phys. C 44 (1989) 441 [inSPIRE].

[5] L. Wolfenstein, Neutrino Oscillations in Matter, Phys. Rev. D 17 (1978) 2369 [InSPIRE].

[6] C. Soumya, M. Ghosh, S.K. Raut, N. Sinha and P. Mehta, Probing muonic charged current nonstandard interactions at decay-at-rest facilities in conjunction with T2HK, Phys. Rev. D 101 (2020) 055009 [arXiv: 1911.05021] [INSPIRE]. 
[7] A.D. Santos, Sensitivity of reactor experiments to nonstandard neutrino interactions in beta decay rates, arXiv:2007.07409 [INSPIRE].

[8] C.X. Yue and X.J. Cheng, Constraints on the charged-current non-standard neutrino interactions induced by the gauge boson W', Nucl. Phys. B 963 (2021) 115280 [arXiv:2008.10027] [INSPIRE].

[9] C. Biggio, M. Blennow and E. Fernandez-Martinez, General bounds on non-standard neutrino interactions, JHEP 08 (2009) 090 [arXiv:0907.0097] [INSPIRE].

[10] S.P. Mikheyev and A.Y. Smirnov, Resonance Amplification of Oscillations in Matter and Spectroscopy of Solar Neutrinos, Sov. J. Nucl. Phys. 42 (1985) 913 [InSPIRE].

[11] T. Ohlsson, Status of non-standard neutrino interactions, Rept. Prog. Phys. 76 (2013) 044201 [arXiv: 1209.2710] [INSPIRE].

[12] A. Chatterjee, F. Kamiya, C.A. Moura and J. Yu, Impact of Matter Density Profile Shape on Non-Standard Interactions at DUNE, arXiv:1809.09313 [INSPIRE].

[13] S.-F. Ge and S.J. Parke, Scalar Nonstandard Interactions in Neutrino Oscillation, Phys. Rev. Lett. 122 (2019) 211801 [arXiv:1812.08376] [InSPIRE].

[14] K.S. Babu, G. Chauhan and P.S. Bhupal Dev, Neutrino nonstandard interactions via light scalars in the Earth, Sun, supernovae, and the early Universe, Phys. Rev. D 101 (2020) 095029 [arXiv: 1912.13488] [INSPIRE].

[15] P. Bakhti and M. Rajaee, Sensitivities of future solar neutrino observatories to nonstandard neutrino interactions, Phys. Rev. D 102 (2020) 035024 [arXiv:2003.12984] [INSPIRE].

[16] A. Garcia, R. Gauld, A. Heijboer and J. Rojo, Complete predictions for high-energy neutrino propagation in matter, JCAP 09 (2020) 025 [arXiv: 2004.04756] [INSPIRE].

[17] N. Fornengo, M. Maltoni, R. Tomas and J.W.F. Valle, Probing neutrino nonstandard interactions with atmospheric neutrino data, Phys. Rev. D 65 (2002) 013010 [hep-ph/0108043] [INSPIRE].

[18] O.G. Miranda, M.A. Tortola and J.W.F. Valle, Are solar neutrino oscillations robust?, JHEP 10 (2006) 008 [hep-ph/0406280] [INSPIRE].

[19] J. Liao, D. Marfatia and K. Whisnant, Degeneracies in long-baseline neutrino experiments from nonstandard interactions, Phys. Rev. D 93 (2016) 093016 [arXiv:1601.00927] [INSPIRE].

[20] P. Coloma and T. Schwetz, Generalized mass ordering degeneracy in neutrino oscillation experiments, Phys. Rev. D 94 (2016) 055005 [Erratum ibid. 95 (2017) 079903] [arXiv: 1604.05772] [INSPIRE].

[21] I. Esteban, M.C. Gonzalez-Garcia, M. Maltoni, I. Martinez-Soler and J. Salvado, Updated constraints on non-standard interactions from global analysis of oscillation data, JHEP 08 (2018) 180 [Addendum ibid. 12 (2020) 152] [arXiv:1805.04530] [INSPIRE].

[22] I. Esteban, M.C. Gonzalez-Garcia and M. Maltoni, On the Determination of Leptonic CP-violation and Neutrino Mass Ordering in Presence of Non-Standard Interactions: Present Status, JHEP 06 (2019) 055 [arXiv: 1905.05203] [INSPIRE].

[23] B. Dutta, R.F. Lang, S. Liao, S. Sinha, L. Strigari and A. Thompson, A global analysis strategy to resolve neutrino NSI degeneracies with scattering and oscillation data, JHEP 09 (2020) 106 [arXiv:2002.03066] [INSPIRE]. 
[24] I. Esteban, M.C. Gonzalez-Garcia and M. Maltoni, On the effect of NSI in the present determination of the mass ordering, arXiv:2004.04745 [INSPIRE].

[25] S. Davidson, C. Pena-Garay, N. Rius and A. Santamaria, Present and future bounds on nonstandard neutrino interactions, JHEP 03 (2003) 011 [hep-ph/0302093] [INSPIRE].

[26] S. Karmakar and S. Pandey, XENON1T constraints on neutrino non-standard interactions, arXiv:2007.11892 [INSPIRE].

[27] KM3NET collaboration, Neutrino Oscillations and Non-standard Interactions with KM3NeT-ORCA, in Prospects in Neutrino Physics, 4, 2020 [arXiv: 2004. 05004] [INSPIRE].

[28] A. Esmaili and A.Y. Smirnov, Probing Non-Standard Interaction of Neutrinos with IceCube and DeepCore, JHEP 06 (2013) 026 [arXiv: 1304.1042] [INSPIRE].

[29] A. Esmaili and H. Nunokawa, On the robustness of IceCube's bound on sterile neutrinos in the presence of non-standard interactions, Eur. Phys. J. C 79 (2019) 70 [arXiv: 1810.11940] [INSPIRE].

[30] M. Masud, A. Chatterjee and P. Mehta, Probing CP-violation signal at DUNE in presence of non-standard neutrino interactions, J. Phys. G 43 (2016) 095005 [arXiv:1510.08261] [INSPIRE].

[31] A. de Gouvêa and K.J. Kelly, Non-standard Neutrino Interactions at DUNE, Nucl. Phys. B 908 (2016) 318 [arXiv:1511.05562] [INSPIRE].

[32] S. Verma and S. Bhardwaj, Nonstandard Interactions and Prospects for Studying Standard Parameter Degeneracies in DUNE and T2HKK, Adv. High Energy Phys. 2019 (2019) 8464535 [arXiv: 1808.04263 ] [INSPIRE].

[33] M. Masud, S. Roy and P. Mehta, Correlations and degeneracies among the NSI parameters with tunable beams at DUNE, Phys. Rev. D 99 (2019) 115032 [arXiv:1812.10290] [INSPIRE].

[34] A. Giarnetti and D. Meloni, Probing Source and Detector NSI parameters at the DUNE Near Detector, arXiv:2005.10272 [INSPIRE].

[35] Super-KAmiokAnde collaboration, Study of Non-Standard Neutrino Interactions with Atmospheric Neutrino Data in Super-Kamiokande I and II, Phys. Rev. D 84 (2011) 113008 [arXiv:1109.1889] [INSPIRE].

[36] BoreXINo collaboration, Constraints on flavor-diagonal non-standard neutrino interactions from Borexino Phase-II, JHEP 02 (2020) 038 [arXiv:1905.03512] [INSPIRE].

[37] N.C. Ribeiro, H. Minakata, H. Nunokawa, S. Uchinami and R. Zukanovich-Funchal, Probing Non-Standard Neutrino Interactions with Neutrino Factories, JHEP 12 (2007) 002 [arXiv:0709.1980] [INSPIRE].

[38] O.G. Miranda and H. Nunokawa, Non standard neutrino interactions: current status and future prospects, New J. Phys. 17 (2015) 095002 [arXiv:1505.06254] [INSPIRE].

[39] L.J. Flores, E.A. Garcés and O.G. Miranda, Exploring NSI degeneracies in long-baseline experiments, Phys. Rev. D 98 (2018) 035030 [arXiv: 1806.07951] [INSPIRE].

[40] W.-J. Feng, J. Tang, T.-C. Wang and Y.-X. Zhou, Nonstandard interactions versus planet-scale neutrino oscillations, Phys. Rev. D 100 (2019) 115034 [arXiv:1909.12674] [INSPIRE]. 
[41] O. Yasuda, Neutrino Oscillations at low energy long baseline experiments in the presence of nonstandard interactions and parameter degeneracy, PTEP 2020 (2020) 063B03 [arXiv: 2002.01616] [INSPIRE].

[42] P.B. Denton, J. Gehrlein and R. Pestes, CP-Violating Neutrino Non-Standard Interactions in Long-Baseline-Accelerator Data, arXiv: 2008.01110 [INSPIRE].

[43] T2K collaboration, Constraint on the matter-antimatter symmetry-violating phase in neutrino oscillations, Nature 580 (2020) 339 [Erratum ibid. 583 (2020) E16] [arXiv: 1910.03887] [INSPIRE].

[44] S.S. Chatterjee and A. Palazzo, Non-standard neutrino interactions as a solution to the

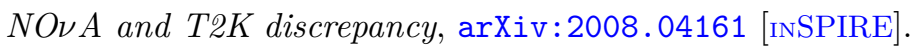

[45] CHARM collaboration, Experimental Verification of the Universality of $\nu_{e}$ and $\nu_{\mu}$ Coupling to the Neutral Weak Current, Phys. Lett. B 180 (1986) 303 [INSPIRE].

[46] NuTeV collaboration, A Precise Determination of Electroweak Parameters in Neutrino Nucleon Scattering, Phys. Rev. Lett. 88 (2002) 091802 [Erratum ibid. 90 (2003) 239902] [hep-ex/0110059] [INSPIRE].

[47] P. Coloma, P.B. Denton, M.C. Gonzalez-Garcia, M. Maltoni and T. Schwetz, Curtailing the Dark Side in Non-Standard Neutrino Interactions, JHEP 04 (2017) 116 [arXiv: 1701.04828] [INSPIRE].

[48] COHERENT collaboration, Observation of Coherent Elastic Neutrino-Nucleus Scattering, Science 357 (2017) 1123 [arXiv: 1708.01294] [INSPIRE].

[49] J. Liao and D. Marfatia, COHERENT constraints on nonstandard neutrino interactions, Phys. Lett. B 775 (2017) 54 [arXiv:1708.04255] [InSPIRE].

[50] P.B. Denton, Y. Farzan and I.M. Shoemaker, Testing large non-standard neutrino interactions with arbitrary mediator mass after COHERENT data, JHEP 07 (2018) 037 [arXiv: 1804.03660] [INSPIRE].

[51] J. Billard, J. Johnston and B.J. Kavanagh, Prospects for exploring New Physics in Coherent Elastic Neutrino-Nucleus Scattering, JCAP 11 (2018) 016 [arXiv: 1805.01798] [INSPIRE].

[52] W. Altmannshofer, M. Tammaro and J. Zupan, Non-standard neutrino interactions and low energy experiments, JHEP 09 (2019) 083 [arXiv: 1812.02778] [INSPIRE].

[53] O.G. Miranda, G. Sanchez Garcia and O. Sanders, Coherent elastic neutrino-nucleus scattering as a precision test for the Standard Model and beyond: the COHERENT proposal case, Adv. High Energy Phys. 2019 (2019) 3902819 [arXiv: 1902.09036] [INSPIRE].

[54] C. Giunti, General COHERENT constraints on neutrino nonstandard interactions, Phys. Rev. D 101 (2020) 035039 [arXiv: 1909.00466] [INSPIRE].

[55] P. Coloma, I. Esteban, M.C. Gonzalez-Garcia and M. Maltoni, Improved global fit to Non-Standard neutrino Interactions using COHERENT energy and timing data, JHEP 02 (2020) 023 [Addendum ibid. 12 (2020) 071] [arXiv: 1911.09109] [INSPIRE].

[56] B.C. Canas, E.A. Garces, O.G. Miranda, A. Parada and G. Sanchez Garcia, Interplay between nonstandard and nuclear constraints in coherent elastic neutrino-nucleus scattering experiments, Phys. Rev. D 101 (2020) 035012 [arXiv:1911.09831] [INSPIRE].

[57] T. Han, J. Liao, H. Liu and D. Marfatia, Nonstandard neutrino interactions at COHERENT, DUNE, T2HK and LHC, JHEP 11 (2019) 028 [arXiv:1910.03272] [INSPIRE]. 
[58] Z. Berezhiani and A. Rossi, Limits on the nonstandard interactions of neutrinos from $e^{+} e^{-}$ colliders, Phys. Lett. B 535 (2002) 207 [hep-ph/0111137] [INSPIRE].

[59] J. Barranco, O.G. Miranda, C.A. Moura and J.W.F. Valle, Constraining non-standard neutrino-electron interactions, Phys. Rev. D 77 (2008) 093014 [arXiv:0711.0698] [INSPIRE].

[60] A. Friedland, M.L. Graesser, I.M. Shoemaker and L. Vecchi, Probing Nonstandard Standard Model Backgrounds with LHC Monojets, Phys. Lett. B 714 (2012) 267 [arXiv:1111.5331] [INSPIRE].

[61] D. Choudhury, K. Ghosh and S. Niyogi, Probing nonstandard neutrino interactions at the LHC Run II, Phys. Lett. B 784 (2018) 248 [arXiv:1801.01513] [INSPIRE].

[62] K.S. Babu, D. Gonçalves, S. Jana and P.A.N. Machado, Neutrino Non-Standard Interactions: Complementarity Between LHC and Oscillation Experiments, arXiv:2003.03383 [INSPIRE].

[63] H.-J. He, Y.-P. Kuang, Y.-H. Qi, B. Zhang, A. Belyaev, R.S. Chivukula et al., CERN LHC Signatures of New Gauge Bosons in Minimal Higgsless Model, Phys. Rev. D 78 (2008) 031701 [arXiv:0708.2588] [inSPIRE].

[64] W. Chao, Z.-G. Si, Z.-z. Xing and S. Zhou, Correlative signatures of heavy Majorana neutrinos and doubly-charged Higgs bosons at the Large Hadron Collider, Phys. Lett. B 666 (2008) 451 [arXiv: 0804.1265] [INSPIRE].

[65] Q.-H. Cao, C.-R. Chen, C.S. Li and H. Zhang, Effective Dark Matter Model: Relic density, CDMS II, Fermi LAT and LHC, JHEP 08 (2011) 018 [arXiv:0912.4511] [INSPIRE].

[66] Y. Bai and T.M.P. Tait, Searches with Mono-Leptons, Phys. Lett. B 723 (2013) 384 [arXiv:1208.4361] [INSPIRE].

[67] N.F. Bell, J.B. Dent, A.J. Galea, T.D. Jacques, L.M. Krauss and T.J. Weiler, Searching for Dark Matter at the LHC with a Mono-Z, Phys. Rev. D 86 (2012) 096011 [arXiv:1209.0231] [INSPIRE].

[68] N. Zhou, D. Berge and D. Whiteson, Mono-everything: combined limits on dark matter production at colliders from multiple final states, Phys. Rev. D 87 (2013) 095013 [arXiv:1302.3619] [INSPIRE].

[69] T. Lin, E.W. Kolb and L.-T. Wang, Probing dark matter couplings to top and bottom quarks at the LHC, Phys. Rev. D 88 (2013) 063510 [arXiv:1303.6638] [INSPIRE].

[70] M. Song, G. Li, W.-G. Ma, R.-Y. Zhang and J.-Y. Guo, Dark matter pair associated with a $W$ boson production at the LHC in next-to-leading order QCD, JHEP 09 (2014) 069 [arXiv: 1403.2142] [INSPIRE].

[71] N.F. Bell, Y. Cai, J.B. Dent, R.K. Leane and T.J. Weiler, Dark matter at the LHC: Effective field theories and gauge invariance, Phys. Rev. D 92 (2015) 053008 [arXiv: 1503.07874] [INSPIRE].

[72] D. Abercrombie et al., Dark Matter Benchmark Models for Early LHC Run-2 Searches: Report of the ATLAS/CMS Dark Matter Forum, Phys. Dark Univ. 27 (2020) 100371 [arXiv: 1507.00966] [INSPIRE].

[73] M. Neubert, J. Wang and C. Zhang, Higher-Order QCD Predictions for Dark Matter Production in Mono-Z Searches at the LHC, JHEP 02 (2016) 082 [arXiv:1509.05785] [INSPIRE]. 
[74] CMS collaboration, Search for supersymmetry in multijet events with missing transverse momentum in proton-proton collisions at 13 TeV, Phys. Rev. D 96 (2017) 032003 [arXiv: 1704.07781] [INSPIRE].

[75] J.A. Aguilar-Saavedra, J.A. Casas, J. Quilis and R. Ruiz de Austri, Multilepton dark matter signals, JHEP 04 (2020) 069 [arXiv: 1911.03486] [INSPIRE].

[76] N. Beni et al., Further studies on the physics potential of an experiment using LHC neutrinos, J. Phys. G 47 (2020) 125004 [arXiv:2004.07828] [INSPIRE].

[77] S. Davidson and V. Sanz, Non-Standard Neutrino Interactions at Colliders, Phys. Rev. D 84 (2011) 113011 [arXiv:1108.5320] [INSPIRE].

[78] Neutrino Non-Standard Interactions: A Status Report SciPost Phys. Proc. 2 (2019) 001 [INSPIRE].

[79] K.S. Babu, P.S.B. Dev, S. Jana and A. Thapa, Non-Standard Interactions in Radiative Neutrino Mass Models, JHEP 03 (2020) 006 [arXiv: 1907.09498] [INSPIRE].

[80] N.F. Bell, Y. Cai and R.K. Leane, Mono-W Dark Matter Signals at the LHC: Simplified Model Analysis, JCAP 01 (2016) 051 [arXiv:1512.00476] [INSPIRE].

[81] Y. Farzan and M. Tortola, Neutrino oscillations and Non-Standard Interactions, Front. in Phys. 6 (2018) 10 [arXiv:1710.09360] [INSPIRE].

[82] J. Heeck, M. Lindner, W. Rodejohann and S. Vogl, Non-Standard Neutrino Interactions and Neutral Gauge Bosons, SciPost Phys. 6 (2019) 038 [arXiv:1812.04067] [InSPIRE].

[83] S. Pandey, S. Karmakar and S. Rakshit, Strong constraints on non-standard neutrino interactions: LHC vs. IceCube, JHEP 11 (2019) 046 [arXiv: 1907.07700] [INSPIRE].

[84] Y. Farzan, A model for lepton flavor violating non-standard neutrino interactions, Phys. Lett. B 803 (2020) 135349 [arXiv: 1912.09408] [INSPIRE].

[85] L.J. Flores, N. Nath and E. Peinado, Non-standard neutrino interactions in U(1)' model after COHERENT data, JHEP 06 (2020) 045 [arXiv:2002.12342] [INSPIRE].

[86] E. Alvarez, M. Estévez and R.M. Sandá Seoane, Z'-explorer: a simple tool to probe Z' models against LHC data, arXiv:2005.05194 [INSPIRE].

[87] D. Buarque Franzosi, M.T. Frandsen and I.M. Shoemaker, New or $\nu$ missing energy: Discriminating dark matter from neutrino interactions at the LHC, Phys. Rev. D 93 (2016) 095001 [arXiv: 1507.07574] [INSPIRE].

[88] M.B. Gavela, D. Hernandez, T. Ota and W. Winter, Large gauge invariant non-standard neutrino interactions, Phys. Rev. D 79 (2009) 013007 [arXiv:0809.3451] [InSPIRE].

[89] T. Han, J. Liao, H. Liu and D. Marfatia, Scalar and tensor neutrino interactions, JHEP 07 (2020) 207 [arXiv:2004.13869] [INSPIRE].

[90] A. Zee, A Theory of Lepton Number Violation, Neutrino Majorana Mass, and Oscillation, Phys. Lett. B 93 (1980) 389 [Erratum ibid. 95 (1980) 461] [InSPIRE].

[91] D. Aristizabal Sierra, M. Hirsch and S.G. Kovalenko, Leptoquarks: Neutrino masses and accelerator phenomenology, Phys. Rev. D 77 (2008) 055011 [arXiv:0710.5699] [INSPIRE].

[92] I. Doršner, S. Fajfer and N. Košnik, Leptoquark mechanism of neutrino masses within the grand unification framework, Eur. Phys. J. C 77 (2017) 417 [arXiv:1701.08322] [InSPIRE].

[93] A. Alloul, N.D. Christensen, C. Degrande, C. Duhr and B. Fuks, FeynRules 2.0 - A complete toolbox for tree-level phenomenology, Comput. Phys. Commun. 185 (2014) 2250 [arXiv: 1310.1921] [INSPIRE]. 
[94] J. Alwall, R. Frederix, S. Frixione, V. Hirschi, F. Maltoni, O. Mattelaer et al., The automated computation of tree-level and next-to-leading order differential cross sections, and their matching to parton shower simulations, JHEP 07 (2014) 079 [arXiv: 1405.0301] [INSPIRE].

[95] T. Sjöstrand, S. Ask, J.R. Christiansen, R. Corke, N. Desai, P. Ilten et al., An introduction to PYTHIA 8.2, Comput. Phys. Commun. 191 (2015) 159 [arXiv:1410.3012] [InSPIRE].

[96] E. Conte, B. Fuks and G. Serret, MadAnalysis 5, A User-Friendly Framework for Collider Phenomenology, Comput. Phys. Commun. 184 (2013) 222 [arXiv:1206.1599] [InSPIRE].

[97] J. Pumplin, D.R. Stump, J. Huston, H.L. Lai, P.M. Nadolsky and W.K. Tung, New generation of parton distributions with uncertainties from global QCD analysis, JHEP 07 (2002) 012 [hep-ph/0201195] [INSPIRE].

[98] ATLAS collaboration, Search for dark matter and other new phenomena in events with an energetic jet and large missing transverse momentum using the ATLAS detector, JHEP 01 (2018) 126 [arXiv:1711.03301] [INSPIRE].

[99] CMS collaboration, Search for new physics in final states with an energetic jet or a hadronically decaying $W$ or $Z$ boson and transverse momentum imbalance at $\sqrt{s}=13 \mathrm{TeV}$, Phys. Rev. D 97 (2018) 092005 [arXiv: 1712.02345] [InSPIRE].

[100] ATLAS collaboration, Search for dark matter in events with a hadronically decaying vector boson and missing transverse momentum in pp collisions at $\sqrt{s}=13 \mathrm{TeV}$ with the ATLAS detector, JHEP 10 (2018) 180 [arXiv:1807.11471] [INSPIRE].

[101] ATLAS collaboration, Search for dark matter at $\sqrt{s}=13 \mathrm{TeV}$ in final states containing an energetic photon and large missing transverse momentum with the ATLAS detector, Eur. Phys. J. C 77 (2017) 393 [arXiv:1704.03848] [INSPIRE].

[102] CMS collaboration, Search for new physics in final states with a single photon and missing transverse momentum in proton-proton collisions at $\sqrt{s}=13 \mathrm{TeV}$, JHEP 02 (2019) 074 [arXiv: 1810.00196] [INSPIRE].

[103] CMS collaboration, Search for high-mass resonances in final states with a lepton and missing transverse momentum at $\sqrt{s}=13 \mathrm{TeV}$, JHEP 06 (2018) 128 [arXiv:1803.11133] [INSPIRE].

[104] ATLAS collaboration, Search for a heavy charged boson in events with a charged lepton and missing transverse momentum from pp collisions at $\sqrt{s}=13 \mathrm{TeV}$ with the ATLAS detector, Phys. Rev. D 100 (2019) 052013 [arXiv: 1906. 05609] [INSPIRE].

[105] M. Cacciari, G.P. Salam and G. Soyez, The anti-k $k_{t}$ jet clustering algorithm, JHEP 04 (2008) 063 [arXiv: 0802.1189] [INSPIRE].

[106] A.L. Read, Presentation of search results: The CL(s) technique, J. Phys. G 28 (2002) 2693 [INSPIRE].

[107] ATLAS collaboration, Search for high-mass dilepton resonances using $139 \mathrm{fb}^{-1}$ of $\mathrm{pp}$ collision data collected at $\sqrt{s}=13 \mathrm{TeV}$ with the ATLAS detector, Phys. Lett. B $\mathbf{7 9 6}$ (2019) 68 [arXiv: 1903.06248] [INSPIRE].

[108] COHERENT collaboration, COHERENT collaboration data release from the first observation of coherent elastic neutrino-nucleus scattering, arXiv:1804.09459 [INSPIRE]. 